Pacific Journal of Mathematics

WHEN CERTAIN NATURAL MAPS ARE EQUIVALENCES 


\section{WHEN CERTAIN NATURAL MAPS ARE EQUIVALENCES}

\section{RICHARD HOLZSAGER}

This paper classifies those spaces for which a certain natural map is a homotopy equivalence. Five cases are considered:

$X \rightarrow S P^{\infty} X$, the map from a space to its infinite symmetric product;

$\Omega^{\infty} S^{\infty} X \rightarrow S P^{\infty} X$, the map from the "infinite loop space of the infinite suspension" to the infinite symmetric product;

$X \rightarrow \Omega^{n} S^{n} X$, the map from a space to the $n$-fold loop space of the $n$-fold suspension;

$S^{n} \Omega^{n} X \rightarrow X$, the map from the $n$-fold suspension of the $n$-fold loop space of a space to the space itself;

$X \rightarrow \Omega^{\infty} S^{\infty} X$, the map from a space to the infinite loop space of the infinite suspension.

Under the assumption (made throughout) that the spaces have the homotopy type of connected $C W$-complexes, these are actually questions about relationships among the homotopy groups, stable homotopy groups and homology groups. The proofs are mostly algebraic.

I. Some properties of $K(Q, 2 k+1)$. Using mapping cylinders, we can construct a sequence $K_{1}, K_{2}, \cdots$ of $C W$-complexes such that each has the homotopy type of the circle, each is a subcomplex of its successor, and the inclusion map of $K_{i}$ into $K_{i+1}$ has degree $i$. Let $K$ be the union of these complexes with the weak topology, i.e. a closed set in $K$ is one whose intersection with each $K_{i}$ is closed in $K_{i}$. Any compact set in $K$ is then contained in one of the $K_{i}$ 's, so the homotopy and homology groups of $K$ are the direct limits of those of the $K_{i}$ 's. It follows that $K$ is of type $K(Q, 1)$ and that $H_{i}(K)$ vanishes for $i$ greater than 1 . I would like now to show in general that $H_{i}(K(Q, 2 k+1))$ vanishes for $i$ greater than $2 k+1$.

First, note that $K(Q, 1)$ has the mod $p$ homology of a point for any prime $p$. It follows by induction, using the spectral sequence of the path fibration over $K(Q, n)$, that the same is true of $K(Q, n)$ for any $n$. This implies, by the Universal Coefficient Theorem, that $H_{*}(K(Q, n))$ is torsion-free. But since the sphere $S^{2 k+1}$ has finite higher homotopy groups, any essential map of $S^{2 k+1}$ into $K(Q, 2+1)$ induces isomorphisms, modulo the class of torsion groups, on homotopy. By Serre's generalized Whitehead theorem, the same is true of homology. Thus, the higher homology of $K(Q, 2 k+1)$ is torsion, and must therefore vanish.

If $X$ is a Moore space of type $M(Q, 2 k+1)(k \geqq 1)$, then by the 
above, a map of $X$ to $K(Q, 2 k+1)$ representing any nonzero element of $H^{2 k+1}(X ; Q)$ induces isomorphisms on all homology groups, and is therefore, by Whitehead's theorems, a homotopy equivalence. Consequently, $X$ is of type $K(Q, 2 k+1)$. In particular, for any positive integer $n$ and any nonnegative integer $k$, the $2 n$-fold suspension $S^{2 n} K(Q, 2 k+1)$ is of type $K(Q, 2(k+n)+1)$.

From the preceding discussion it follows that a space $X$ of type $K(Q, 2 k+1)$ has the following properties:

1. The Hurewicz homomorphisms from $\pi_{i}(X)$ to $H_{i}(X)$ are isomorphisms for all positive integers $i$.

2. The stable Hurewicz homomorphisms from the stable homotopy groups $\pi_{i}^{s}(X)$ to $H_{i}(X)$ are isomorphisms for all $i$.

3. The $2 n$-fold suspension homomorphism from $\pi_{i}(X)$ to $\pi_{i+2 n}\left(S^{2 n} X\right)$ are isomorphisms for all $i$ and $n$.

4. The $2 n$-fold homology suspension homomorphisms from $H_{i}\left(\Omega^{2 n} X\right)$ to $H_{i+2 n}(X)$ (where $\Omega$ denotes the loop-space functors) are isomorphisms for all $i$ (provided $n$ is not greater than $k$ ).

5. The canonical homomorphisms from $\pi_{i}(X)$ to $\pi_{i}^{s}(X)$ are isomorphisms for all positive $i$.

The following sections will be devoted to the classification of spaces with these properties. All spaces are assumed to have homotopy type of connected $C W$-complexes.

II. Hurewicz homomorphisms and stable Hurewicz homomorphisms.

LEMma 1. If a group $G$ has nontrivial torsion, then either $H_{r+2(p-1)}(K(G, r))$ or $H_{r+2 p-1}(K(G, r))$ is nonzero ( $p$ any prime for which $G$ has torsion, $r$ any positive integer).

Proof. Under the hypothesis there exists, for some prime $p$, a monomorphism of $Z_{p}$ (the integers $\bmod p$ ) into $G$. The corresponding map from $K\left(Z_{p}, r\right)$ to $K(G, r)$ then induces an epimorphism of $H^{r+1}\left(K(G, r) ; Z_{p}\right)$ onto $H^{r+1}\left(K\left(Z_{p}, r\right) ; Z_{p}\right)$, by the Universal Coefficient Theorem and right exactness of the functor Ext. In particular, if $i$ is the fundamental class in $H^{r}\left(K\left(Z_{p}, r\right) ; Z_{p}\right)$ and $b$ is the Bockstein coboundary operator associated to the sequence $0 \rightarrow Z_{p} \rightarrow Z_{p^{2}} \rightarrow Z_{p} \rightarrow 0$, then $b(i)$ is in the image, so the lemma will follow if it is shown that the Steenrod reduced power operation $P^{1}$ (which is understood to be $S q^{2}$ if $p=2$ ) acts nontrivially on $b(i)$. By universality of $i$, it therefore suffices to prove that $P^{1} b(x)$ is nonzero for some $r$-dimensional mod $p$ cohomology class $x$ on some space. But if we choose for $x$ the 
$r$-fold cross product $z \times z \times \cdots \times z \times z$, where $z$ is a generator of $H^{1}\left(K\left(Z_{p}, 1\right) ; Z_{p}\right)$, then $p^{1} b(x)$ is the alternating sum of the terms $z \times \cdots \times z \times(b(z))^{p} \times z \times \cdots \times z$ which is nonzero by the Künneth theorem.

Lemma 2. If the group $G$ is not divisible, then either

$$
H_{r+2(p-1)}(K(G, r+1)) \quad \text { or } \quad H_{r+2 p-1}(K(G, r+1))
$$

is nonzero (for any positive) $r$ and any prime for which $Z_{p} \otimes G \neq 0$ ).

Proof. Since $G$ is not divisible there exists, for some prime $p$, an epimorphism of $G$ onto $Z_{p}$. A homomorphism of $Z$ to $G$ can therefore be chosen so that the composite map of $Z$ to $Z_{p}$ is onto. From the induced diagram

$$
H^{r+1}\left(K\left(Z_{p}, r+1\right) ; Z_{p}\right) \rightarrow H^{r+1}\left(K(G, r+1) ; Z_{p}\right) \rightarrow H^{r+1}\left(K(Z, r+1) ; Z_{p}\right)
$$

it then follows that the second homomorphism is onto. The lemma will therefore be proved if we can exhibit an $(r+1)$-dimensional mod $p$ cohomology class on some space which is a reduced integral class and on which $P^{1}$ acts nontrivially. But if $x$ is as in the proof of Lemma 1 , then $b(x)$ certainly has these properties.

LEMMA 3. If $K(G, r)$ has no homology above dimension $r$ (and $G$ is nonzero), then $r$ is odd and the rank of $G$ (i.e. the dimension of the rational vector space $G \otimes Q$ ) is one.

Proof. Since $G$ is nonzero and, by Lemma 1 , torsion-free, there exists a monomorphism of $Z$ into $G$. The corresponding homomorphism of $H^{r}(K(G, r) ; Q)$ to $H^{r}(K(Z, r) ; Q)$ is then onto. When $r$ is even, the generator of the second group has nonzero cup square, which leads to a contradiction of the hypothesis. If the rank of $G$ were greater than one, there would exist monomorphisms of $Z \times Z$ into $G$. The corresponding maps from $H^{r}(K(G, r) ; Q)$ to $H^{r}(K(Z, r) \times K(Z, r) ; Q)$ would then be onto. But by the Künneth theorem there are nontrivial cup products in the latter space, again leading to a contradiction.

Theorem 1. All the Hurewicz maps of a space $X$ are isomorphisms if and only if $X$ is either of type $K(Q, 2 k+1)$ or of type $K(G, 1)$ for some subgroup $G$ of $Q$.

Proof. That $K(Q, 2 k+1)$ has this property was noted in $\S I$. Since any nonzero subgroup $G$ of $Q$ can be represented as the direct limit of a sequence of monomorphisms of $Z$ into itself, $K(G, 1)$ can be 
built as $K(Q, 1)$ was in $\S I$, as the union of subcomplexes with the homotopy type of the circle. Thus, $K(G, 1)$ has the desired property.

Conversely, suppose $X$ has this property. For each positive integer $i$ there is a map $f_{i}$ from $X$ to $K\left(H_{i}(X), i\right)$ which induces the identity on $H_{i}$ (by the Universal Coefficient Theorem). Let $K$ be the weakly topologized union of the spaces $K\left(H_{1}(X), 1\right) \times \cdots \times K\left(H_{r}(X), r\right)$, with the $r$-fold product imbedded in the $(r+1)$-fold product by choosing a base-point in $K\left(H_{r+1}(X), r+1\right)$. We may assume that $f_{i}$ takes the $(i-1)$-skeleton of $X$ to the base point of $K\left(H_{i}(X), i\right)$, and there is then an induced map from $X$ to $K$. The hypothesis on $X$ together with the choice of the maps $f_{i}$ implies that this map induces isomorphisms on all homotopy groups, hence also on all homology groups. By the Künneth theorem it results that none of the $K\left(H_{i}(X), i\right)$ can have any homology above dimension $i$, and that for $i \neq j$ the group $H_{i}(X) \otimes H_{j}(X)$ (and also Tor $\left(H_{i}(X), H_{j}(X)\right)$ must be zero. Since the tensor product of two nonzero torsion-free groups is always nonzero, these conditions, together with the lemmas, finish the proof.

Theorem 2. The stable Hurewicz homomorphisms for a space $X$ are all isomorphisms if and only if $H_{i}(X)$ is torsion-free and divisible for every positive integer $i$.

Proof. First let us show that the second condition implies the first. It was seen in $\S I$ that the stable Hurewicz homomorphisms are isomorphisms if $X$ is the Moore space $M(Q, 2 k+1)$. Since both homology and stable homotopy are generalized homology theories, the same holds for $M(Q, 2 k+2)=S M(Q, 2 k+1)$. Since both functors take arbitrary one point unions to direct sums, it also holds for $M(V, i)$ where $V$ is any vector space over $Q$ (i.e. any torsion-free divisible group). Now let $X$ be any space with torsion-free divisible homology. By suspending, we may as well assume that $X$ is simply connected. We shall show by induction on the difference between the integer $r$ and the connectivity of $X$ that the stable Hurewicz homomorphism in dimension $r$ is an isomorphism. If this difference is one, the Hurewicz theorem gives the result. Suppose the difference is greater than one, and let $X$ be $(i-1)$-connected. Using the Hurewicz theorem, we may find a map $f$ from $M=M\left(H_{i}(X), i\right)$ to $X$ inducing the identity on $H_{i}$. Let $C$ be the mapping cone of $f$. $C$ is $i$-connected and we have the following commutative diagram with both rows exact:

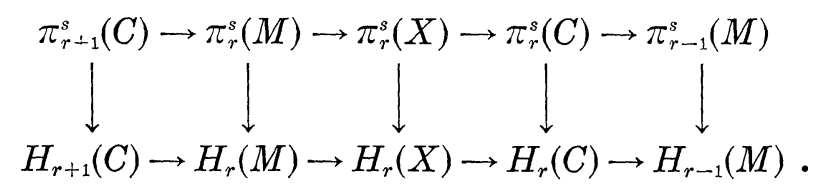


We already know that the second and fifth vertical maps are isomorphisms. By the inductive hypothesis, so is the fourth. One half of the five lemma now implies that the third map is onto. But the difference on which we are inducting is the same for the first map as for the third, so the first must also be onto. The other half of the five lemma finishes this part of the proof.

Now suppose that all stable Hurewicz homomorphisms for $X$ are isomorphisms. Then $S^{r+2 p} X$ has the property that its (ordinary) Hurewicz homomorphisms are isomorphisms up to dimension $2 r+2(p-1)$ and an epimorphism in dimension $2 r+2 p-1$. Thus, as in the proof of Theorem 1, we may find a map from $S^{r+2 p} X$ to

$$
K\left(H_{1}(X), r+2 p+1\right) \times \cdots \times K\left(H_{r+2 p-1}(X), 2 r+4 p-1\right)
$$

which is an isomorphism on homotopy groups up to dimension $2 r+4 p-1$. The same then holds for homology by the Whitehead theorem, which implies that $K\left(H_{r}(X), 2 r+2 p\right)$ has no homology in dimensions $2 r+$ $4 p-3,2 r+4 p-2$, or $2 r+4 p-1$. By Lemmas 1 and 2 , this finishes the proof.

\section{Suspension homomorphisms and their stabilizations.}

THEOREM 3. The n-fold suspension homomorphism from $\pi_{r}(X)$ to $\pi_{r+n}\left(S^{n} X\right)$ is an isomorphism for every $r$ ( $n$ a fixed positive integer) if and only if either $X$ is contractible or $X=K(Q, 2 k+1)$ and $n$ is even.

Proof. The implication in one direction was seen in $\S I$. Suppose then that $X$ has the property in question. This is equivalent to saying that the natural map from $X$ to $\Omega^{n} S^{n} X$ induces isomorphisms on all homotopy groups. Consequently this map induces isomorphisms on cohomology in all dimensions over any coefficient group. But these maps on cohomology are left inverses to the cohomology suspension homomorphisms from $H^{r+n}\left(S^{n} X ; G\right)$ to $H^{r}\left(\Omega^{n} S^{n} X ; G\right)$ (identifying the first of these with $\left.H^{r}(X ; G)\right)$. From the relationship between the transgression operator and the cohomology suspension it follows, since we have just shown the latter to be onto, that every element of $H^{r}\left(\Omega^{n} S^{n} X ; G\right)$ is transgressive with respect to the spectral sequence of the path fibration over $\Omega^{n-1} S^{n} X$, and in fact transgresses all the way up to $H^{r+n}\left(S^{n} X ; G\right)$. Note also that since all these fibrations have contractible total spaces, the transgressions are monomorphisms. Therefore, assuming henceforth that $G$ is a field, it follows from the fact that the differentials of a spectral sequence are derivations with respect to the product structure, that the product of two linearly independent transgressive elements cannot be transgressive. Thus, 
the total reduced cohomology ring of $\Omega^{n} S^{n} X$ over $G$ (and therefore also that of $X$ ) must be at most one-dimensional. Further, if $G$ has characteristic other than 2 , the square of a nonzero even dimensional transgressive element cannot be transgressive, so, in this case, any nontrivial cohomology must occur in an odd dimension.

What we have shown so far is that over any coefficient field $G$, the cohomology ring of $\Omega^{n} S^{n} X$ (and of $X$ ) is either trivial or is an exterior algebra on one generator $x$, and the dimension $r$ of $x$ is odd if $G$ is not of characteristic 2. In the case where the cohomology ring is an exterior algebra, it follows from the spectral sequence that the cohomology ring of $\Omega^{n-1} S^{n} X$ is the polynomial algebra generated by the transgression $t(x)$. Now let $G$ be the prime field $Z_{p}$. Then if $i=(r+1) / 2$ and $P^{i}$ is the Steenrod operation $\left(S_{q}^{2 i}\right.$ if $\left.p=2\right)$, we have $P^{i} t(x)=(t(x))^{p} \neq 0$. As already noted, transgression is monomorphic in the cases we are considering, so $P^{i} t^{n}(x)=t^{n-1} P^{i} t(x)$ is nonzero. But this contradicts the fact that $H^{n+r+i}\left(S^{n} X ; Z_{p}\right)$, being isomorphic to $H^{r+i}\left(X ; Z_{p}\right)$, is trivial. Consequently, $H^{*}\left(X ; Z_{p}\right)$ must be trivial.

The only remaining possibilities (by the Universal Coefficient Theorem) are that the homology of $X$ is trivial, or else consists of one odd dimensional group isomorphic to $Q$. In the first case $X$ is contractible, since $\pi_{1}(X)=\pi_{n+1}\left(S^{n} X\right)$ is abelian. In the second case, it follows by induction that $H^{*}\left(\Omega^{n-s} S^{n} X ; Q\right)$ is either an exterior algebra or a polynomial algebra depending on whether $s$ is even or odd. But for $s=n$ we must get an exterior algebra, so $n$ has to be even. Then $S^{n} X$ is a Moore space $M(Q, n+r)$, and also an EilenbergMacLane space $K(Q, n+r)$ by $\S I, n+r$ being odd. $\Omega^{n} S^{n} X$, and therefore also, $X$, must then be of type $K(Q, r)$, which completes the proof.

Corollary (Theorem 4). If $X$ is simply connected, then the homology suspension homomorphism from $H_{r}\left(\Omega^{n} X\right)$ to $H_{r+n}(X)$ is an isomorphism for every positive integer $r$ ( $n$ a fixed positive integer) if and only if either $X$ is contractible or $X$ is of type $K(Q, 2 k+1)$ with $n=2 i$ for some $i$ not exceeding $k$.

Proof. The implication in one direction was seen in $\S I$. Conversely, the condition on the homology suspension means that the natural map from $S^{n} \Omega^{n} X$ to $X$ induces isomorphisms on homology: Since both spaces are simply connected, the Whitehead theorems imply that this map is a homotopy equivalence. Therefore, so is the induced map from $\Omega^{n}\left(S^{n} \Omega^{n} X\right)$ to $\Omega^{n}(X)$. But this map is a left inverse to the natural map from $\Omega^{n} X$ to $\Omega^{n} S^{n}\left(\Omega^{n} X\right)$. The latter map must then also be a homotopy equivalence. The result now follows easily from an 
application of Theorem 2 to $\Omega^{n} X$.

THEOREM 5. The canonical homomorphism from $\pi_{r}(X)$ to $\pi_{r}^{s}(X)$ is an isomorphism for every positive integer $r$ if and only if $X$ is either contractible or of type $K(Q, 2 k+1)$.

Proof. The implication in one direction was seen in $\S I$. The Proof in the other direction is only a modification of the proof of Theorem 3. Indeed, the condition of this theorem is equivalent to the condition that the natural map from $X$ to $\Omega^{n} S^{n} X$ induce isomorphisms on homotopy groups in dimensions up to $n$, for every integer $n$. The arguments of Theorem 3 imply, in this case, first that the dimension of the total reduced cohomology ring of $X$ up to dimension $n / 2$ over any coefficient field $G$ is at most one, occurring in an odd dimension if $G$ has characteristic other than 2, and second that there is no nontrivial mod $p$ cohomology up to dimension $(n / p)-1$ for any prime $p$. Since these facts hold for any integer $n$, it follows from the Universal Coefficient Theorem that $X$ has the homology of a point or of a $K(Q, 2 k+1)$. By Theorem 2 it has the right stable homotopy, and by the hypothesis of this theorem, the right homotopy.

Received May 24, 1971. This paper is part of the author's thesis, prepared under the direction of R. Bott. 



\section{PACIFIC JOURNAL OF MATHEMATICS}

\section{EDITORS}

H. SAMELSON

Stanford University

Stanford, California 94305

C. R. HobBY

University of Washington

Seattle, Washington 98105
J. DUGUNDJI

Department of Mathematics

University of Southern California

Los Angeles, California 90007

RICHARD ARENS

University of California

Los Angeles, California 90024

\section{ASSOCIATE EDITORS}

E. F. BECKENBACH

B. H. NeumanN

F WoLF

K. YoshidA

\section{SUPPORTING INSTITUTIONS}

UNIVERSITY OF BRITISH COLUMBIA

UNIVERSITY OF SOUTHERN CALIFORNIA

CALIFORNIA INSTITUTE OF TECHNOLOGY

UNIVERSITY OF CALIFORNIA

MONTANA STATE UNIVERSITY

STANFORD UNIVERSITY

UNIVERSITY OF NEVADA

NEW MEXICO STATE UNIVERSITY

OREGON STATE UNIVERSITY

UNIVERSITY OF OREGON

OSAKA UNIVERSITY

UNIVERSITY OF TOKYO

UNIVERSITY OF UTAH

WASHINGTON STATE UNIVERSITY

UNIVERSITY OF WASHINGTON

$\stackrel{*}{*} \stackrel{*}{*} \stackrel{*}{*}{ }^{*}{ }^{*}$ MMEICAN MATHEMATICAL SOCIETY

NAVAL WEAPONS CENTER

Printed in Japan by International Academic Printing Co., Ltd., Tokyo, Japan 


\section{Pacific Journal of Mathematics}

\section{Vol. 42, No. $1 \quad$ January, 1972}

Tage Bai Andersen, On Banach space valued extensions from split faces ........

David Marion Arnold, A duality for quotient divisible abelian groups of finite

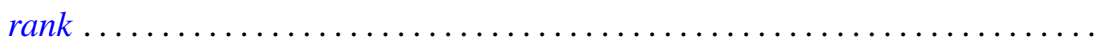

Donald Pollard Ballou, Shock sets for first order nonlinear hyperbolic

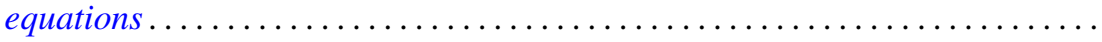

Leon Brown and Lowell J. Hansen, On the range sets of $H^{p}$ functions .........

Alexander Munro Davie and Arne Stray, Interpolation sets for analytic

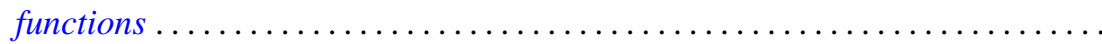

M. G. Deshpande, Structure of right subdirectly irreducible rings. II . . . . . . . . .

Barry J. Gardner, Some closure properties for torsion classes of abelian

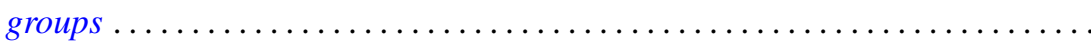

Paul Daniel Hill, Primary groups whose subgroups of smaller cardinality are

direct sums of cyclic groups . . . . . . . . . . . . . . . . . . .

Richard Allan Holzsager, When certain natural maps are equivalences .........

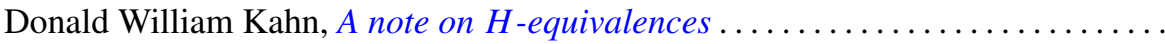

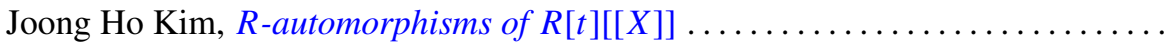

Shin'ichi Kinoshita, On elementary ideals of polyhedra in the 3-sphere.........

Andrew T. Kitchen, Watts cohomology and separability...

Vadim Komkov, A technique for the detection of oscillation of second order

ordinary differential equations .

Charles Philip Lanski and Susan Montgomery, Lie structure of prime rings of characteristic 2

Andrew Lenard, Some remarks on large Toeplitz determinants . .

Kathleen B. Levitz, A characterization of general Z.P.I.-rings. II .

Donald A. Lutz, On the reduction of rank of linear differential systems

David G. Mead, Determinantal ideals, identities, and the Wronskian ...

Arunava Mukherjea, A remark on Tonelli's theorem on integration in product

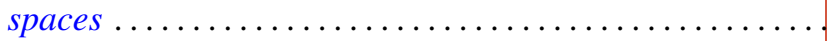

Hyo Chul Myung, A generalization of the prime radical in nonassociative rings.

John Piepenbrink, Rellich densities and an application to unconditionally nonoscillatory elliptic equations.

Michael J. Powers, Lefschetz fixed point theorems for a new class of multi-valued maps .

Aribindi Satyanarayan Rao, On the absolute matrix summability of a Fourier

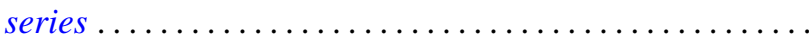

T. S. Ravisankar, On Malcev algebras ......................... 227

William Henry Ruckle, Topologies on sequences spaces . . . . . . . . . . . . . 235

Robert C. Shock, Polynomial rings over finite dimensional rings . . . . . . . . . 251

Richard Tangeman, Strong heredity in radical classes . . . . . . . . . . . . . . 259

B. R. Wenner, Finite-dimensional properties of infinite-dimensional spaces . . . . 267 